\title{
Protocol for the development of a Core Outcome Set (COS) for hemorrhoidal disease: an international Delphi study
}

\author{
R. R. van Tol ${ }^{1}$ J. Melenhorst ${ }^{1}$ - C. D. Dirksen ${ }^{2}$ L. P. S. Stassen ${ }^{1} \cdot$ S. O. Breukink ${ }^{1}$
}

Accepted: 28 April 2017 / Published online: 13 May 2017

(C) The Author(s) 2017. This article is an open access publication

\begin{abstract}
Purpose Over the last decade, many studies were performed regarding treatment options for hemorrhoidal disease. Randomised controlled trials (RCTs) should have welldefined primary and secondary outcomes. However, the reported outcome measures are numerous and diverse. The heterogeneity of outcome definition in clinical trials limits transparency and paves the way for bias. The development of a core outcome set (COS) helps minimizing this problem. A COS is an agreed minimum set of outcomes that should be measured and reported in all clinical trials of a specific disease. The aim of this project is to generate a COS regarding the outcome of treatment after hemorrhoidal disease.

Methods A Delphi study will be performed by an international steering group healthcare professionals and patients with the intention to create a standard outcome set for future clinical trials for the treatment of hemorrhoidal disease. First, a literature review will be conducted to establish which outcomes are used in clinical trials for hemorrhoidal disease. Secondly, both healthcare professionals and patients will participate in several consecutive rounds of online questionnaires and a face-to-face meeting to refine the content of the COS.
\end{abstract}

Electronic supplementary material The online version of this article (doi:10.1007/s00384-017-2833-5) contains supplementary material, which is available to authorized users.

R. R. van Tol

robin.van.tol@mumc.nl

1 Department of Surgery, Maastricht University Medical Centre, P. Debelaan 25, 6229 HX Maastricht, the Netherlands

2 Department of Clinical Epidemiology and Medical Technology Assessment, Maastricht University Medical Centre, P. Debyelaan 25, 6229 HX Maastricht, the Netherlands
Discussion Development of a COS for hemorrhoidal disease defines a minimum outcome-reporting standard and will improve the quality of research in the future.

Keywords Core outcome set · Delphi · Endpoints . Hemorrhoids $\cdot$ Anal $\cdot$ Protocol

\section{Background}

Hemorrhoidal disease is one of the most common anorectal disorders presenting to coloproctological units, with a prevalence of $5-35 \%$ in the overall population $[1,2]$. The true incidence of hemorrhoidal disease is difficult to estimate, as many patients are reluctant to seek medical therapy for various personal, cultural, and socioeconomic reasons. Hemorrhoids are enlarged vascular cushions in the anal canal, which can bleed because of both degenerative effects of aging and the repeated passage of hard stool [1]. People experience the following most common complaints: blood loss, prolapse, pain, itching, and pruritus. Conservative and/or medical treatment, including diet, lifestyle changes, and application of topical ointments, is often offered first [3]. When these treatment options fail, a common next treatment step is rubber band ligation (RBL) [4, 5]. Grade III and IV hemorrhoidal disease is often directly treated by more invasive surgical interventions including the sutured hemorrhoidopexy [6], the stapled hemorrhoidopexy [7,8], the Doppler-guided hemorrhoidal artery ligation (DGHAL) [9-11], and the traditional hemorrhoidectomy $[12,13]$.

The ability to compare findings between studies and synthesize data in meta-analysis is limited because the outcomes are inconsistently defined and reported in clinical trials. This hampers interpreting treatment effect and making evidencebased healthcare decisions $[14,15]$. 
The Outcome Measures in Rheumatology (OMERACT) [16] and Core Outcome Measures in Effective Trials (COMET) [17] (http://www.comet-initiative.org/) initiatives responded to the problem by developing a guideline for the creation of a core outcome set (COS).

This paper describes the study protocol developing a COS that should be considered mandatory for inclusion in all future clinical trials on treatment of hemorrhoidal disease.

\section{Methods/design}

Development of a COS incorporates the Delphi methodology and exists of a stepwise approach [18]. The guideline on the usage of the Delphi technique and the checklist will be followed [19]. First, a systematic literature review will be performed to identify potential outcomes. Secondly, several rounds of online questionnaires will be conducted involving researchers and healthcare professionals.

\section{Search strategy}

A broad-search strategy will be conducted to identify all published guidelines, reviews, systematic reviews, meta-analyses, and protocols regarding hemorrhoidal disease. The search will be limited to English language articles published in full-text. Searches will be performed in the following database: PubMed, EMBASE, and Cochrane between January 2012 and December 2016 (Appendix 1).

\section{Selection of articles}

The review will be performed by two researchers (RT and JM) to identify all possible clinical outcomes reported in treatment studies for hemorrhoidal disease. First, the identified abstract will be screened. Studies where the outcomes are not treatment-related (e.g. health policy) will be excluded. In order to ensure accuracy of exclusion, a proportion of the excluded abstracts will be reviewed by a third reviewer (SB).

\section{Data extraction}

For each trial, we will assess the author, date of publication, study design, compared treatments/interventions, primary and secondary outcomes, definition of these outcomes, and patient-reported outcomes (PROs).

\section{Participants}

We will involve healthcare professionals and patients. We found no guidelines for the sample sizes of this study [18, 20]. However, having more stakeholders will increase the reliability of the group judgment $[19,20]$. Healthcare professionals will be selected from a diverse range of international institutions and organizations. We will select healthcare professionals who have multiple cited publications in the field of proctology and/or who are familiar with the development of a COS. Further, we will use the "snowball sampling" approach. This method gives the healthcare professionals the opportunity to add someone they want to be included as panel member. We aim to recruit around 30 healthcare professionals by e-mail.

Patients will be invited to participate when they visit the outpatient clinic for the first time. Participants have to be diagnosed with hemorrhoidal disease and have to agree to participate in all Delphi rounds needed for this COS. We expect to recruit about 20 patients in total.

\section{Online survey}

The list of outcomes, originating from the systematic review, will be formatted into questions with a Likert response design $[21,22]$, an evaluation scale from 1 to 9 , to identify outcomes of importance to both healthcare professionals and patients. Questionnaires in the Delphi survey will be pilot tested by at least two members of the steering group (SB and LS).

Currently, there is no consensus in literature regarding the cut offs for inclusion and exclusion of outcomes in this process. Therefore, a flexible and pragmatic approach will be employed. "Consensus in" (information essential to the core set) will be defined as greater than $70 \%$ of participants scoring as 7-9 and less than $15 \%$ of participants scoring as $1-3$. "Consensus out" (information should not be included in core set) will be defined as greater than $70 \%$ of participants scoring as 1-3 and less than $15 \%$ of participants scoring as $7-9$. "Disagreement" will occur when $33 \%$ or more score $1-3$ and $33 \%$ or more score 7-9 for a particular item. All other combinations will be considered "Equivocal," which means that these items are open to more than one interpretation [23].

Over the course of several rounds, the expectation is that the range of items will decrease and the group converges toward a consensus opinion. This part of the process will end when the list of items is reduced to ten or less; or on completion of the second round voting [Appendix 2].

\section{Round 1}

In the first round, the panel members will be approached by a personalized e-mail with a link to a web-based survey (SurveyMonkey). Once participants have registered for the survey, names and email addresses will be stored in the system. This will allow identification of participants completing all rounds of the Delphi survey.

Initially, the panel members will be provided with one page background information on the rationale of the development of the COS. Then, they will be asked to list all items that they 
consider important or relevant in the COS. All items included in Delphi round 1 will be assigned to one of the four categories and "consensus in" and "consensus out" domains will be brought forward for the second round. Items designated "Disagreement" will undergo further analysis: mean scores will be calculated, and if the mean is above or below 5 (i.e., tending toward "consensus in" or "consensus out"), the item will be included in the second round. Items designated "Equivocal" will not be carried forward.

\section{Round 2}

The panel members from round 1 will then be invited to undertake round 2 of the process. During the second round, the stakeholders will be asked to review their initial responses in the light of groups' responses [Appendix 3]. The responses of round 1 will be aggregated and send back to the panel members anonymously in a feedback report. The participants do not know the identities of other individuals in the panel group to make sure that the views of participants are obtained by a method that gives equal influence. This report will include the panel members' ratings on the several questions/items, including the median scores, the interquartile ranges, their comments, and suggestions. Descriptive statistics summarizing the results of round 1 will be available for the panel members to review [Appendix 4]. Participants will also be provided with an option to add additional items that they think are missing.

\section{Round 3}

The third round will only be undertaken if significant numbers of outcomes remain in the short list after round 2 . The results of round 2 will again be send back to all panel members in a feedback report. Then, the panel members will be asked to rerate the items in view of the groups' response.

\section{Consensus meeting}

If consensus has not been reached after the three Delphi rounds or there is significant disagreement between the stakeholders, we will conduct a face-to-face meeting with the panel members initially invited.

By the end of the process, we should have identified "what" COS should be measured in clinical trials for hemorrhoidal disease. However, we may not be clear on "how" we should measure these.

\section{Discussion}

The aim of this project is the development of a COS for hemorrhoidal disease. At the time of writing, there is no published
COS for hemorrhoidal disease. The development of COS for the management of hemorrhoidal disease will facilitate evidence synthesis by reducing heterogeneity between trials.

The Delphi method $[18,24]$ is the appropriate instrument in decision-making processes in groups as feedback can be provided in a controlled anonymous manner. A major drawback is that the results of a Delphi study are highly dependent upon the composition of the panel and the selection of its members. To provide the highest possible input, we will include the healthcare professionals from a diverse range of international institutions and organizations.

We must actively engage with trial-lists and those that fund and publish trials to ensure that our COS is used in the rest of the academic world. With help from the panel members, we will identify how to reach relevant audience, including people using services, carers, the public, practitioners, and providers.

\section{Trial status}

The Delphi study is currently ongoing. We are preparing to recruit participants to the Delphi study. The final COS is expected by the end of 2017 .

Authors' contributions Robin R. van Tol, M.D.: Acquisition, analysis and interpretation of data, drafting of the article, final approval of article.

Jarno Melenhorst, M.D., Ph.D.: Analysis and interpretation of data, drafting of the article, final approval of article.

Carmen D. Dirksen, Ph.D., Prof.: Conception and design, interpretation of data, revision and final approval of article.

Laurents P. Stassen, M.D., Ph.D., Prof.: Conception and design, interpretation of data, revision and final approval of article.

Stephanie O. Breukink, M.D., Ph.D.: Conception and design, interpretation of data, revision and final approval of article.

\section{Compliance with ethical standards}

Ethics approval The ethical committee of Maastricht University Medical Centre confirmed that the Medical Research Involving Human Subjects Act (WMO) does not apply to the abovementioned study and that an official approval of this study by the committee is not required (METC 16-4-078).

Open Access This article is distributed under the terms of the Creative Commons Attribution 4.0 International License (http:// creativecommons.org/licenses/by/4.0/), which permits unrestricted use, distribution, and reproduction in any medium, provided you give appropriate credit to the original author(s) and the source, provide a link to the Creative Commons license, and indicate if changes were made.

\section{References}

1. Loder PB, Kamm MA, Nicholls RJ, Phillips RK (1994) Haemorrhoids: pathology, pathophysiology and aetiology. Br J Surg 81(7):946-954

2. Lohsiriwat V (2012) Hemorrhoids: from basic pathophysiology to clinical management. World J Gastroenterol 18(17):2009-2017 
3. Alonso-Coello P, Mills E, Heels-Ansdell D, Lopez-Yarto M, Zhou Q, Johanson JF et al (2006) Fiber for the treatment of hemorrhoids complications: a systematic review and meta-analysis. Am J Gastroenterol 101(1):181-188

4. Brown S, Tiernan J, Biggs K, Hind D, Shephard N, Bradburn M et al (2016) The HubBLe trial: haemorrhoidal artery ligation (HAL) versus rubber band ligation (RBL) for symptomatic second- and third-degree haemorrhoids: a multicentre randomised controlled trial and health-economic evaluation. Health Technol Assess 20(88): $1-150$

5. Gaj F, Biviano I, Sportelli G, Candeloro L (2015) Rubber band ligation in treatment of hemorrhoids: our experience. Clin Ter 166(4):162-167

6. Pakravan F, Helmes C, Baeten C (2009) Transanal open hemorrhoidopexy. Dis Colon rectum 52(3):503-506

7. Corsale I, Rigutini M, Francioli N, Panicucci S, Mori PA, Aloise F (2014) Stapled anopexy and STARR in surgical treatment of haemorrhoidal disease. Updat Surg 66(3):217-222

8. Nisar PJ, Acheson AG, Neal KR, Scholefield JH (2004) Stapled hemorrhoidopexy compared with conventional hemorrhoidectomy: systematic review of randomized, controlled trials. Dis Colon rectum 47(11): 1837-1845

9. Faucheron JL, Poncet G, Voirin D, Badic B, Gangner Y (2011) Doppler-guided hemorrhoidal artery ligation and rectoanal repair (HAL-RAR) for the treatment of grade IV hemorrhoids: longterm results in 100 consecutive patients. Dis Colon rectum 54(2): 226-231

10. Hoyuela C, Carvajal F, Juvany M, Troyano D, Trias M, Martrat A et al (2016) HAL-RAR (Doppler guided haemorrhoid artery ligation with recto-anal repair) is a safe and effective procedure for haemorrhoids. Results of a prospective study after two-years follow-up. Int J Surg 28:39-44

11. Zagriadskii EA [Transanal Doppler-guided desarterization with mucopexy (HAL-RAR) for the treatment of hemorrhoids stage III-IV]. Khirurgiia (Mosk). 2013(4):59-64

12. Shanmugam V, Thaha MA, Rabindranath KS, Campbell KL, Steele RJ, Loudon MA (2005) Systematic review of randomized trials comparing rubber band ligation with excisional haemorrhoidectomy. Br J Surg. 92(12):1481-1487

13. Watson AJ, Hudson J, Wood J, Kilonzo M, Brown SR, McDonald A et al (2016) Comparison of stapled haemorrhoidopexy with traditional excisional surgery for haemorrhoidal disease (eTHoS): a pragmatic, multicentre, randomised controlled trial. Lancet 388(10058):2375-2385

14. Clarke M (2008) Standardising outcomes in Paediatric clinical trials. PLoS Med 5(4):e102

15. Boonen A, Stucki G, Maksymowych W, Rat AC, Escorpizo R, Boers M et al (2009) The OMERACT-ICF reference group: integrating the ICF into the OMERACT process: opportunities and challenges. J Rheumatol 36(9):2057-2060

16. Boers M, Brooks P, Strand CV, Tugwell P (1998) The OMERACT filter for outcome measures in rheumatology. J Rheumatol 25(2): 198-199

17. Prinsen CA, Vohra S, Rose MR, King-Jones S, Ishaque S, Bhaloo Z et al (2014) Core outcome measures in effectiveness trials (COMET) initiative: protocol for an international Delphi study to achieve consensus on how to select outcome measurement instruments for outcomes included in a 'core outcome set'. Trials 15:247

18. Steurer J (2011) The Delphi method: an efficient procedure to generate knowledge. Skelet Radiol 40(8):959-961

19. Sinha IP, Smyth RL, Williamson PR (2011) Using the Delphi technique to determine which outcomes to measure in clinical trials: recommendations for the future based on a systematic review of existing studies. PLoS Med 8(1):e1000393

20. Boers M, Kirwan JR, Wells G, Beaton D, Gossec L, d'Agostino MA et al (2014) Developing core outcome measurement sets for clinical trials: OMERACT filter 2.0. J Clin Epidemiol 67(7):745-753

21. Ogden J, Lo J (2012) How meaningful are data from Likert scales? An evaluation of how ratings are made and the role of the response shift in the socially disadvantaged. J Health Psychol 17(3):350-361

22. van Laerhoven H, van der Zaag-Loonen HJ, Derkx BH (2004) A comparison of Likert scale and visual analogue scales as response options in children's questionnaires. Acta Paediatr 93(6):830-835

23. Schunemann HJ, Mustafa R, Brozek J, Santesso N, Alonso-Coello P, Guyatt $G$ et al (2016) GRADE guidelines: 16. GRADE evidence to decision frameworks for tests in clinical practice and public health. J Clin Epidemiol 76:89-98

24. Boulkedid R, Abdoul H, Loustau M, Sibony O, Alberti C (2011) Using and reporting the Delphi method for selecting healthcare quality indicators: a systematic review. PLoS One 6(6):e20476 